\title{
Chest adipose tissue distribution in patients with morbid obesity
}

\author{
Adam Lemanowicz ${ }^{\mathrm{A}, \mathrm{B}, \mathrm{C}, \mathrm{D}, \mathrm{E}, \mathrm{F}}$, Waldemar Leszczyński ${ }^{\mathrm{C}, \mathrm{D}, \mathrm{E}}$, Grażyna Rusak ${ }^{\mathrm{B}, \mathrm{D}, \mathrm{E}}$, Marcin Białecki, ${ }^{\mathrm{B}, \mathrm{D}, \mathrm{E}}$, \\ Przemysław Ratajczak ${ }^{\mathrm{D}, \mathrm{F}}$
}

Department of Radiology and Diagnostic Imaging, Nicolaus Copernicus University in Toruń, Collegium Medicum in Bydgoszcz, Bydgoszcz, Poland

\section{Abstract}

Purpose: Obesity is a well-known of risk factor for atherosclerosis and the amount of visceral adipose tissue is considered as an independent predictor of coronary artery disease (CAD). An aim of the study was to investigate the distribution of intrathoracic adipose tissue in morbidly obese patients.

Material and methods: Fifty-one patients with morbid obesity (BMI $\geq 40 \mathrm{~kg} / \mathrm{m}^{2}$ ) and thirty controls were scanned in a coronary calcium scoring protocol. Control group consisted of patients scanned due to a clinical suspicion of CAD, who did not fulfill obesity criteria. The amount of adipose tissue was measured as epicardial adipose tissue (EAT) thickness, pericoronary fat (PCF) thickness, total intra-pericardial fat (IPF) volume, and total intrathoracic fat (ITF) volume.

Results: Mean BMI of obese patients and controls was 47.3 and 26.5 , respectively $(p<0.0001)$. Patients with obesity and controls did not differ with respect to mean EAT, mean PCF, and IPF. However, ITF was lower in obesity group than in control group ( 268 vs. $332 \mathrm{~cm}^{3}$, respectively; $p<0.03$ ). Moreover, ROC analysis presented relation between obesity and the superior EAT thickness, PCF at LCX, mean PCF, ITF, and chest soft tissue (CST) thickness $(p<0.03)$. CST thickness of $>60 \mathrm{~mm}$ was the parameter that presented the strongest association with morbid obesity (AUC $0.95 ; p<0.0001$ ).

Conlcusions: Increased chest soft tissue thickness but not the increased intrathoracic adipose tissue volume was associated with morbid obesity. Since the quantity of the pericardiac fat is not directly related to the obesity, its accumulation may be related to a mechanism different than that of subcutaneous adipose tissue growth.

Key words: adipose tissue, multidetector computed tomography, obesity, morbid.

\section{Introduction}

According to the WHO definition, obesity is a pathological accumulation of fat in the adipose tissue beyond physiological needs and adaptability, which may lead to adverse health effects. The worldwide prevalence of obesity more than doubled between 1980 and 2014. Overall, about 13\% of the world's adult population (11\% of men and $15 \%$ of women) were obese in 2014, which amounted to approximately 600 million individuals [1].

Because obesity is a risk factor for many significant disorders, it has become a major concern in recent decades.
This is particularly noticeable in highly developed countries, where a large percentage of the population is affected by overweight and obesity and where most of their negative consequences emerge. Obesity comorbidities, including dyslipidemia, non-insulin-dependent diabetes mellitus and hypertension, are risk factors of atherosclerosis eventually leading to coronary heart disease (CHD) or stroke. Excessive adiposity is also related to certain types of cancer, gallbladder disease, osteoarthritis, gout and pulmonary diseases, including sleep apnea [2].

The development of obesity may be triggered by various factors, e.g. endocrine diseases, use of certain medications, mental disorders or genetic factors. But in most

Correspondence address:

Adam Lemanowicz, Department of Radiology and Diagnostic Imaging, Nicolaus Copernicus University in Toruń, Collegium Medicum in Bydgoszzz,

9 Skłodowskiej-Curie St., 85-094 Bydgoszzz, Poland, e-mail: adam.lemanowizz@cm.umk.pl

Authors' contribution:

A Study design · B Data collection · C Statistical analysis · D Data interpretation - E Manuscript preparation · F Literature search · G Funds collection 
cases, the underlying reason is an excessive food intake combined with sedentary lifestyle. They both lead to a positive energy balance and finally to weight gain. Overweight and obese individuals differ not only in the amount of fat but also in its regional distribution. The main types of obesity are "android obesity" (associated with intra-abdominal fat accumulation) and the "gynoid obesity", in which fat is distributed more evenly, peripherally around the body. Nowadays, visceral adipose tissue (VAT) is considered as an active endocrine gland, secreting i.a. proinflammatory mediators. These factors may induce chronic inflammation and oxidative stress, which are believed to contribute to the development and growth of atherosclerotic plaques. Some trials have already confirmed that excessive visceral, abdominal fat is associated with accelerated progression of atherosclerosis and higher cardiovascular disease burden $[3,4]$. However, an increasing number of studies suggest that intra-thoracic fat (ITF) may exaggerate the progression of coronary atherosclerosis $[5,6]$. ITF, also referred to as pericardial adipose tissue (PAT), is classified as visceral fat and consists of epicardial and para-cardiac fat layers, separated by the pericardium. Although epicardial adipose tissue (EAT) and para-cardiac adipose tissue are thought to differ anatomically, embriologically, physiologically, biomolecularly, and clinically [7], the emerging evidence has indicated that they both are equally metabolically active. These findings suggest that toxic effects exerted by ITF depots on coronary arteries might be particularly significant in obese people.

It is recognized that android obesity poses a much greater health risk than the less serious gynoid obesity $[8,9]$. This relation is explained mainly by the fact that subcutaneous adipose tissue (SAT) is biologically less active than abdominal fat. Moreover, it has been discovered that removing a large amount of subcutaneous fat by liposuction does not improve the CHD metabolic risk factors associated with abdominal obesity [10]. Probably that is why relatively little attention is paid to the subcutaneous adipose tissue. However, in patients with morbid obesity, its quantity may be large enough that it may also contribute to negative consequences such as insulin resistance [11]. Furthermore, new discoveries allowed to hypothesize about the existence of the so-called "obesity paradox". It was already recognized in some cases of obesity, where moderate obesity apparently increased the survival rate in certain diseases (chronic kidney disease, congestive heart failure, chronic obstructive pulmonary disease, coronary artery disease) [12]. Explanation for this phenomenon may be that the type and location of adipose tissue and not the total amount of the body fat constitute a risk factor of those diseases. Indeed, recent studies show that in healthy subjects increased amounts of subcutaneous fat have a protective effect by inhibiting the development of atherosclerosis [13]. The phenomenon of obesity paradox encourages further research, particularly in the context of linking it to clusters of fat in specific areas of the body.
While the amount of intra-abdominal adipose tissue can be easily estimated by body mass index (BMI) combined with the waist-hip ratio, it is more difficult to determine the amount of fat deposited within the thoracic cavity. The aim of our study was to investigate the quantity and distribution of thoracic adipose tissue in morbidly obese patients using computed tomography.

\section{Material and methods}

\section{Patients}

A study group of 51 subjects, including 44 women and 7 men, with morbid obesity $\left(B M I \geq 40 \mathrm{~kg} / \mathrm{m}^{2}\right)$ was recruited from patients scheduled for bariatric surgery in our hospital. Exclusion criteria were: body weight over $220 \mathrm{~kg}$ (scanner's table limit), inability to fit the scanner's gantry, dyspnea in supine position, previous coronary stenting or coronary surgery, arrhythmia or tachycardia, pregnancy, age below 18 years.

Due to possible ethical concerns, a control group of 30 subjects was composed retrospectively from patients who were scanned due to a clinical suspicion of coronary artery disease and who did not fulfill obesity criteria. Exclusion criteria were similar as in the study group.

\section{Imaging methods}

All subjects underwent an ECG-gated cardiac CT scanning according to the coronary calcium scoring protocol using a 64-row Philips Brilliance unit $[14,15]$. No intravenous contrast media nor premedication were used. Standard Philips Brilliance Workstation was used to evaluate a number of morphological parameters for the quantification of body fat. The quantity of epicardial fat was expressed as EAT thickness and peri-coronary fat thickness (PCF) as well as intra-pericardial fat (IPF) volume. The total amount of fat inside the chest was measured as ITF volume. Due to limited FOV in our CS protocol, a rough estimate of subcutaneous fat was determined in a simplified manner on the basis of the CT scout view.

Epicardial fat thickness was measured on oblique MPR images. Reconstruction planes were located at the base of the ventricles and adjusted to be perpendicular to the long axis of the heart. Calipers were placed at the 25\% (EAT sup), 50\% (EAT mid) and 75\% (EAT inf) of the right ventricle's wall, between myocardium and the visceral epicardium and perpendicular to the surface of the heart (Figure 1). The mean value of these measurements was also used in statistical analysis.

Pericoronary fat thickness was measured on standard axial scans. Calipers were placed in the regions of right coronary artery (PCF RCA), left coronary artery (PCF LCA) and the left circumflex (PCF LCX). The maximal distance between myocardium and the visceral epicardium was measured. To avoid overestimation of PCF thickness 


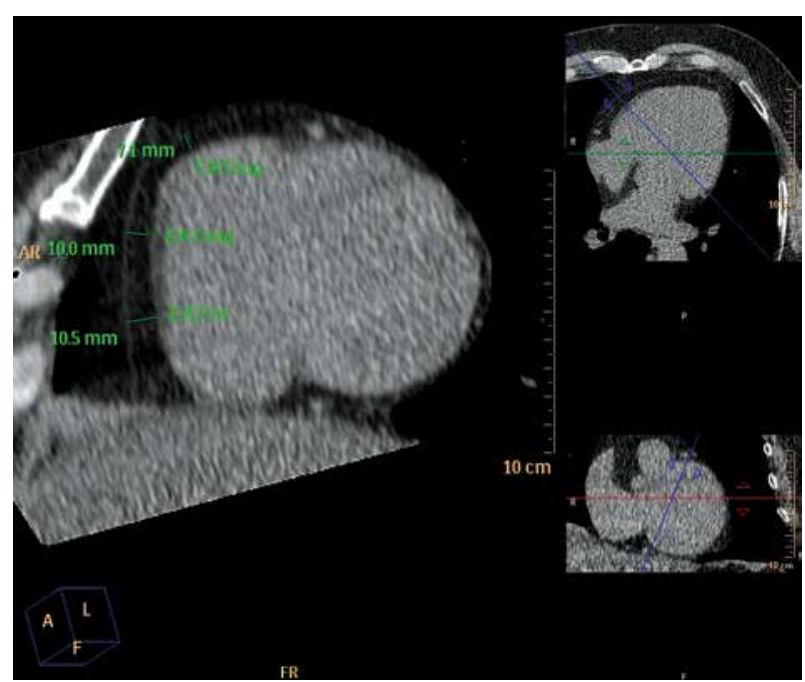

Figure 1. Measurement of the epicardial adipose tissue thickness (EAT). Reconstruction plane was adjusted to be perpendicular to the long axis of the heart and located at the base of the ventricles. Calipers were placed at the $25 \%$ (EAT sup), $50 \%$ (EAT mid) and 75\% (EAT inf) of the right ventricle's free wall, between myocardium and the visceral epicardium and perpendicular to the surface of the heart

due to obliquity, measurements were performed only on sections where surface of the heart was perpendicular to the slice plane. The mean value of these measurements was also used in statistical analysis (Figure 2).

In order to isolate voxels of adipose tissue for a volumetric quantification, a density range from -200 to $-30 \mathrm{HU}$ was used. Intra-pericardial fat volume (IPF vol) (Figure 3) and intra-thoracic fat volume (ITF vol) (Figure 4) were calculated automatically after adequate fat depots were manually outlined slice-by-slice on axial scans. Tracing of the pericardium and the mediastinal boundary began at the level of pulmonary artery bifurcation and ended with the last slice on which pericardium was recognizable (IPF) or at the surface of the diaphragm (ITF).

Finally, we used CT scout views to measure total chest width and the distance between external outlines of ribs, slightly above the right dome of the diaphragm. The difference of these two measurements divided by 2 gave the approximate thickness of chest soft tissues (CST thickness) (Figure 5).

\section{Statistical analysis}

Statistical analysis was performed using MedCalc v. 14 (MedCalc Software bvba, NL). Normality of the data was tested using the Kolmogorov-Smirnov test. Differences in quantitative data were tested using the independent samples $t$-test. Comparison of qualitative data was performed using the Chi-squared test. $P$-value of 0.05 was considered statistically significant. ROC statistics and logistic regression analysis were used to detect the relation between obesity and the above-mentioned morphometric parameters.

\section{Results}

Both groups did not differ significantly in terms of gender and age distribution. Subjects from the study group were 19 to 69 years old (mean 44.1), while the age of controls ranged from 30 to 71 years (mean 54.1). Mean BMI of obese patients and controls was 47.3 and 26.5 , respectively $(p<0.0001)$. The results of chest fat quantitative assessment in both groups are summarized in Table 1 .

Patients with obesity and controls did not differ with respect to mean EAT, mean PCF and IPF volume. Only epicardial adipose tissue thickness at the middle level of the right ventricle's front wall and the thickness of fat
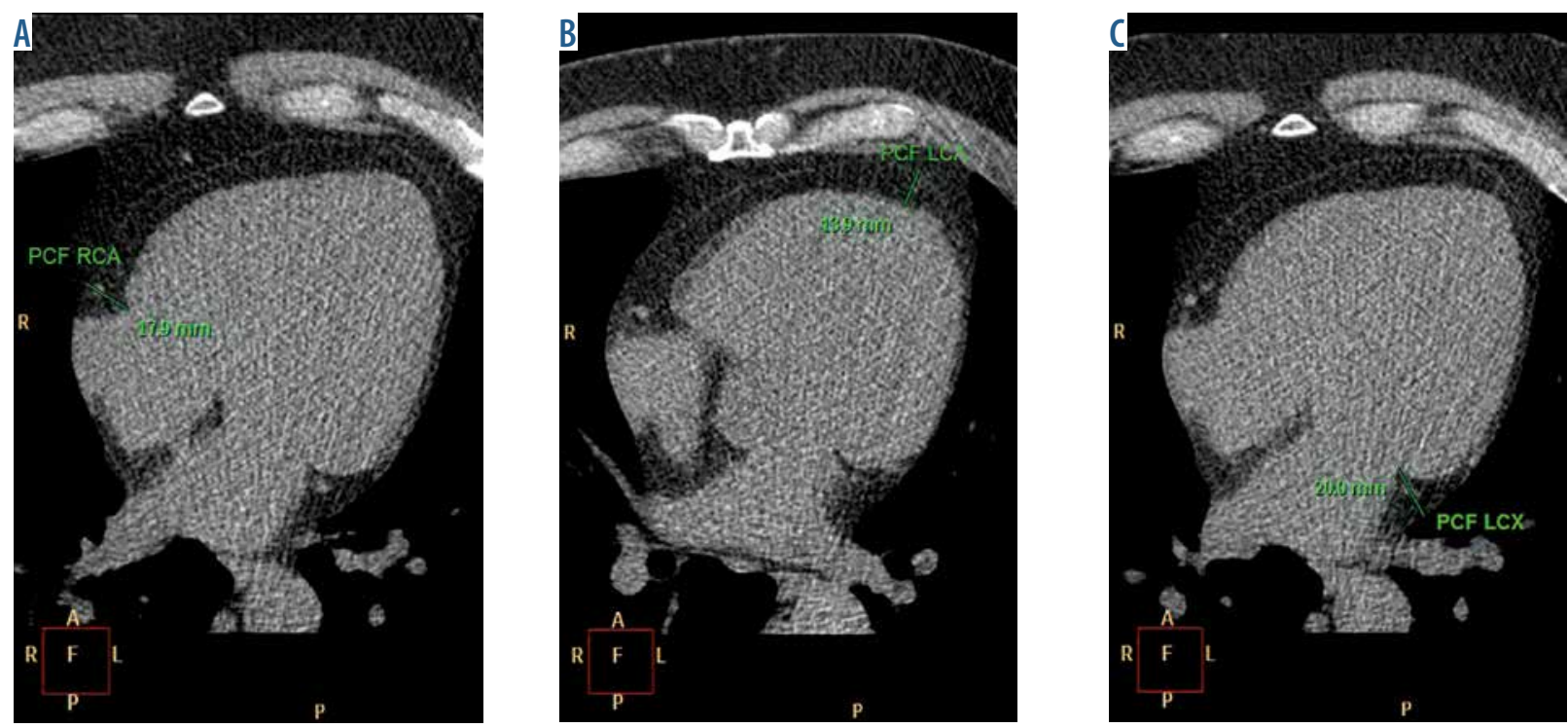

Figure 2. Measurement of the pericoronary fat thickness (PCF). Standard axial view is used. Calipers were placed in the regions of right coronary artery (PCF RCA), left coronary artery (PCF LCA) and the left circumflex (PCF LCX). Maximal distance between myocardium and the visceral epicardium was measured. To avoid overestimation of PCF thickness due to obliquity, measurements were done only on sections where surface of the heart was perpendicular to the slice plane 


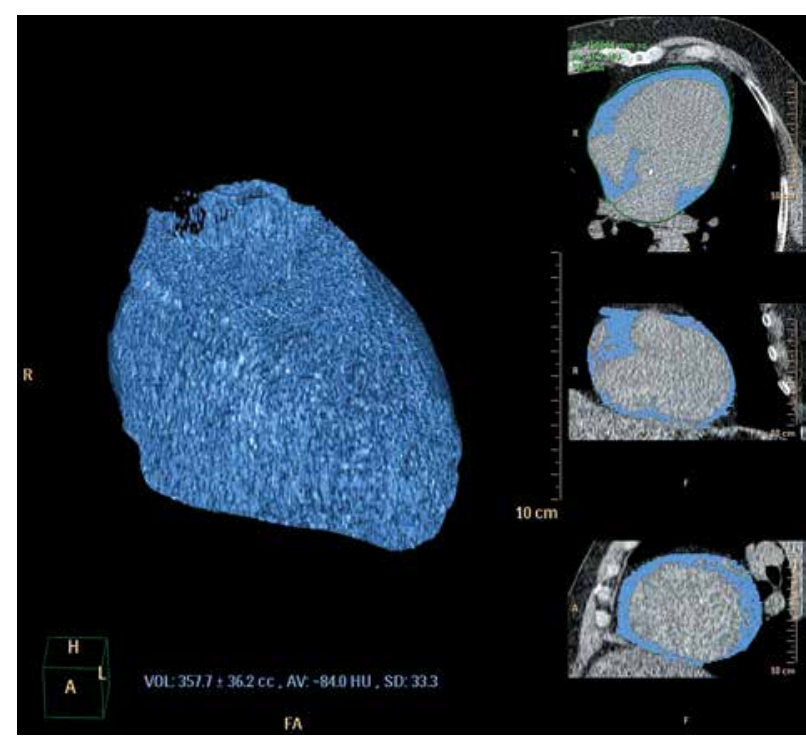

Figure 3. Measurement of the intra-pericardial fat volume (IPF vol). Results obtained by manual, slice-by-slice tracing of the pericardium on axial sections, beginning at the level of pulmonary artery bifurcation and ending with the last slice on which pericardium was recognizable. Attenuation values from $-200 \mathrm{HU}$ to $-30 \mathrm{HU}$ were used to identify fat voxels

over the circumflex branch of left coronary artery were significantly higher in the study group than in the control group. However, ITF volume was lower in the obesity group than in the control group (268 vs. $332 \mathrm{~cm}^{3}$, respectively; $p<0.03$ ) (Table 2).

Furthermore, ROC analysis presented the relation between obesity and the superior EAT thickness, PCF around LCX, mean PCF, ITF and chest soft tissue thickness $(p<0.03)$ (Table 3$)$. CST thickness of $>60 \mathrm{~mm}$ was the parameter that presented the strongest association with morbid obesity (AUC $0.95 ; p<0.0001$ ) (Figure 6). In the logistic regression analysis, CST thickness was the only independent predictor of obesity with an odds ratio of 1.22 (95\% CI: $1.04-1.42 ; p=0.0144)$.

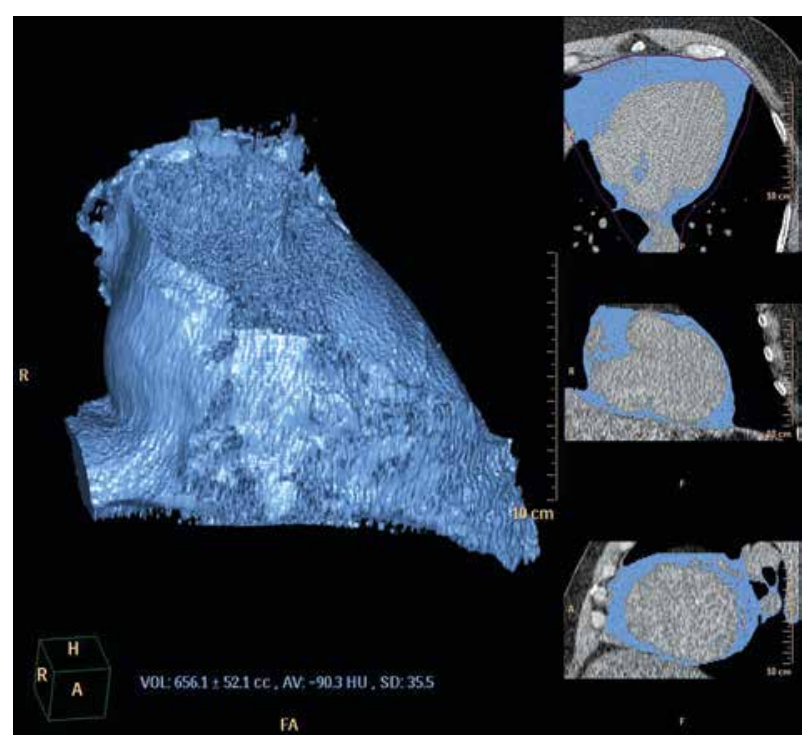

Figure 4. Measurement of the intra-thoracic fat volume (ITF vol). Results obtained by manual, slice-by slice tracing of the mediastinum boundary on axial sections, beginning at the level of pulmonary artery bifurcation and ending at the surface of the diaphragm. Attenuation values from $-200 \mathrm{HU}$ to $-30 \mathrm{HU}$ were used to identify fat voxels

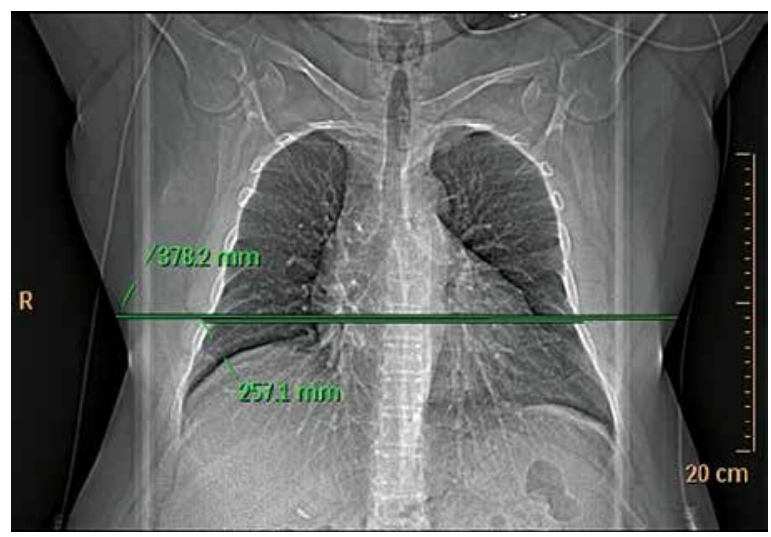

Figure 5. Method of the approximate measurement of the CST thickness on the CT scout view

Table 1. Results of adipose tissue quantitative analysis and chest morphology measurements

\begin{tabular}{|c|c|c|c|c|c|c|}
\hline & \multicolumn{3}{|c|}{ Study group } & \multicolumn{3}{|c|}{ Control group } \\
\hline & $\begin{array}{c}\text { Mean value } \\
(95 \% \text { Cl) }\end{array}$ & $\begin{array}{l}\text { Minimal } \\
\text { value }\end{array}$ & $\begin{array}{l}\text { Maximal } \\
\text { value }\end{array}$ & $\begin{array}{l}\text { Mean value } \\
(95 \% \mathrm{Cl})\end{array}$ & $\begin{array}{l}\text { Minimal } \\
\text { value }\end{array}$ & $\begin{array}{l}\text { Maximal } \\
\text { value }\end{array}$ \\
\hline EAT inf $(\mathrm{mm})$ & $5.80(5.04-6.55)$ & 1.2 & 14.0 & $5.15(4.41-5.89)$ & 2.5 & 9.7 \\
\hline EAT mid (mm) & $4.57(3.92-5.22)$ & 0.5 & 14.0 & $4.75(3.89-5.61)$ & 0.0 & 10.0 \\
\hline EAT sup (mm) & $5.03(4.43-5.62)$ & 1.0 & 12.8 & $4.05(3.40-4.71)$ & 1.6 & 8.7 \\
\hline EAT mean (mm) & $5.13(4.50-5.76)$ & 0.9 & 13.6 & $4.65(4.02-5.27)$ & 2.3 & 8.3 \\
\hline PCF LCA (mm) & $7.31(6.67-7.95)$ & 2.6 & 13.1 & $11.47(10.67-12.26)$ & 7.9 & 17.4 \\
\hline PCF LCX (mm) & $12.57(11.93-13.21)$ & 8.7 & 18.6 & $6.87(6.07-7.66)$ & 2.3 & 10.5 \\
\hline PCF RCA (mm) & $15.04(14.21-15.87)$ & 7.0 & 22.9 & $14.52(13.57-15.48)$ & 9.6 & 19.0 \\
\hline PCF mean (mm) & $11.64(11.05-12.23)$ & 7.3 & 18.2 & $10.95(10.30-11.61)$ & 7.5 & 15.3 \\
\hline IPF vol $\left(\mathrm{cm}^{3}\right)$ & $126.00(109.00-145.00)$ & 45 & 380.0 & $131.00(108.00-155.00)$ & 40.0 & 252.0 \\
\hline ITF vol $\left(\mathrm{cm}^{3}\right)$ & $268.00(236.00-300.00)$ & 99 & 664.0 & $332.00(284.00-381.00)$ & 123.0 & 748.0 \\
\hline CST thickness (mm) & $81.10(72.80-89.40)$ & 21.5 & 117.0 & $30.2(24.7-35.7)$ & 6.0 & 59.5 \\
\hline
\end{tabular}


Table 2. Comparison of the amount of fat around the heart between the study group and the control group with respective levels of statistical significance of the difference

\begin{tabular}{|l|c|}
\hline & $p$-value \\
\hline EAT sup & 0.0376 \\
\hline EAT mid & 0.7336 \\
\hline EAT inf & 0.2509 \\
\hline EAT mean & 0.3097 \\
\hline PCF LCA & 0.3376 \\
\hline PCF LCX & 0.0326 \\
\hline PCF RCA & 0.3744 \\
\hline PCF mean & 0.1181 \\
\hline IPF vol & 0.7556 \\
\hline ITF vol & 0.0225 \\
\hline
\end{tabular}

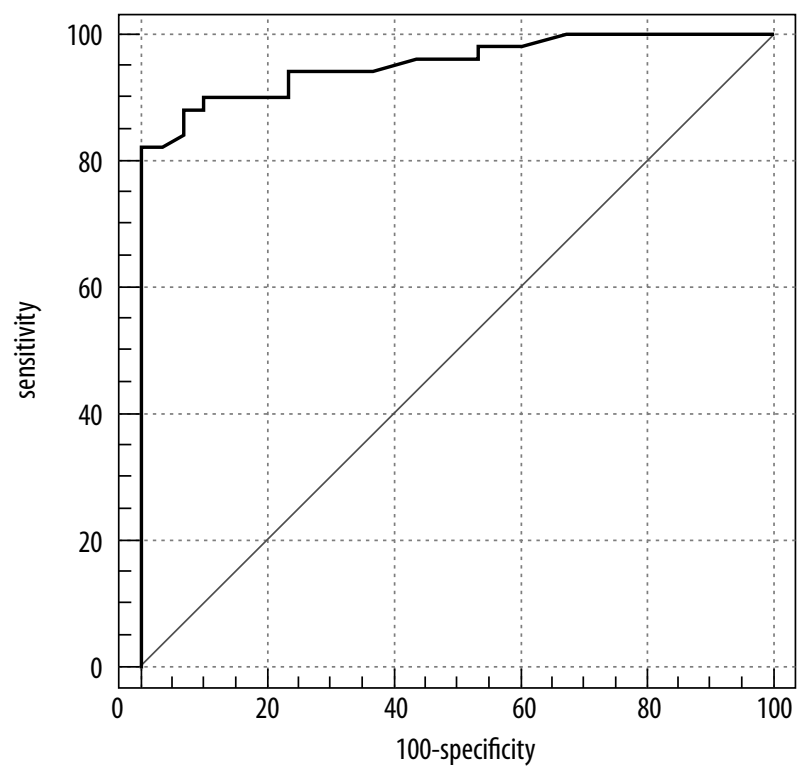

Figure 6. The ROC curve for the relationship between obesity and the chest soft tissues thickness in all patients

Table 3. Analysis of the ROC curves for the relationship between obesity and the evaluated morphological parameters in all patients. Only statistically significant data are presented

\begin{tabular}{|l|c|c|c|c|c|} 
& AUC $(95 \% \mathrm{Cl})$ & $p$-value & Threshold & Sensitivity & Specificity \\
\hline EAT sup & $0.65(0.53-0.75)$ & 0.0239 & $>4.7$ & 56 & 70 \\
\hline ITF vol & $0.66(0.54-0.76)$ & 0.0180 & $\leq 298.7$ & 73 & 67 \\
\hline PCF LCX & $0.65(0.53-0.75)$ & 0.0200 & $>12.5$ & 50 & 77 \\
\hline PCF mean & $0.70(0.58-0.79)$ & 0.0012 & $>11.2$ & 70 & 66 \\
\hline CST thickness & $0.95(0.88-0.99)$ & $<0.0001$ & $>60.0$ & 82 & 100 \\
\hline
\end{tabular}

\section{Discussion}

Our study showed that the amount of ITF is not directly related to obesity. Interestingly, the total ITF volume in morbidly obese patients appeared to be lower than that of patients with a suspicion of CAD. Moreover, chest soft tissue thickness determined on CT scout view was the only independent predictor of obesity.

As CST thickness is easy to assess, it might prove to be useful in clinical practice. However, it would require a prior confirmation of its association with, for example, cardiovascular risk factors. Also, it represents a noteworthy observation because most obesity studies focus on the evaluation of visceral fat. Our study, in turn, showed that in patients with morbid obesity the amount of subcutaneous fat may be subject to proportionally greater growth. This observation requires further studies, since the available literature is insufficient in this area and brings different conclusions. For example, in the Framingham Heart Study, both BMI and waist circumference were strongly correlated with SAT and VAT after adjustment for age [16]. Whereas Prado et al. showed that patients with metabolic syndrome have significantly higher waist circumference, BMI, visceral fat thickness, preperitoneal circumference but not subcutaneous fat thickness, as compared to healthy individuals [17].
As regards ITF deposits, previous studies have shown that the thickness of epicardial adipose tissue of the right ventricle, as assessed by echocardiography, is correlated with waist circumference, the amount of visceral fat of the abdomen, the values of diastolic blood pressure, insulin levels in serum, fasting insulin resistance and the severity of coronary artery disease [18-20]. It seems, however, that CT due to its high spatial resolution, as compared to echocardiography, should be a better modality for a reliable quantification of pericardial fat. An additional advantage of this method was that it was used in this study with ECG triggering. The triggering is a standard option in the calcium scoring protocol, and significantly reduces artifacts related to heart motion $[14,15]$. As shown by Gorter et al., measurements of fat volume by computed tomography have good reproducibility and a low coefficient of variation ranging from $3.0 \%$ to $5.0 \%$. In contrast, only moderate repeatability of measurement was established for measuring the thickness of EAT in CT (CV of 11.0-23.4\%) [21].

Our study, involving patients with morbid obesity, indicates that the amount of para-cardiac adipose is not directly related to obesity. This seems to confirm other mechanisms of subcutaneous and visceral fat accumulation. The ROC curve analysis showed the relationship between obesity and the thickness of epicardial fat meas- 
ured at the level of the upper $25 \%$ of the RV anterior wall (EAT sup), a medium thickness of pericoronary fat (PCF mean), the thickness of fat around the LCX, the volume of ITF and also with the width of the soft tissues of the chest. Interestingly, the ITF volume was significantly higher in the control group. The explanation for this observation seems to be quite difficult. It may indicate that the process of accumulating ITF in patients with morbid obesity occurs with a lower intensity than in non-obese patients. An alternative explanation would require a theory of severe accumulation of thoracic fat in patients with coronary atherosclerotic burden. It should be noted that the control group consisted of patients with a clinical indication for coronary CT angiography, and that they presented a significantly higher prevalence of other cardiovascular risk factors such as hypertension, smoking and dyslipidemia. This is an interesting observation, as it has been previously postulated that the accumulation of adipose tissue around the heart may provide a compensating mechanism for myocardium that would otherwise be more vulnerable to ischemia. The basis for such a hypothesis is the finding that infiltration of inflammatory cells, such as macrophages to the adipose tissue, can promote angiogenesis [22]. In other studies, various dependencies were detected. Mahabadi et al. analyzed the volume of fat in 3 locations (pericardial fat, ITF and visceral abdominal fat), which showed a close correlation with each other [23]. Liu et al., using data from the Jackson Heart Study, found that the amount of pericardial fat significantly correlated with BMI and the abdominal VAT [6]. Similarly, Gorter et al. reported that the amount of the epicardial adipose tissue was found to be associated with obesity [21]. Greif et al. found a significant increase in the volume of "pericardial fat" (PAT, approximately corresponding to ITF parameter in this paper) with age [24]. A similar correlation was observed by Jeong et al. [20]. Moreover, in the study by Greif, female patients had a lower volume of pericardial fat compared to men irrespective of age [24]. Moreover, in a scientific evaluation of 3312 participants from the Framingham Heart Study by Thanassoulis et al., the average volume of pericardial fat $(\mathrm{PFV})$ was higher in men $\left(117.5 \mathrm{~cm}^{3}\right)$ than in women $\left(93.9 \mathrm{~cm}^{3}\right)$ [25]. In the Yong's study, pericardial fat area on CT sections correlated with age, HDL-cholesterol and the area occupied by the visceral fat on abdominal CT scans [26].

On the other hand Rosenquist et al. examined visceral and subcutaneous fat not quantitatively, but qualitatively. They showed that the attenuation coefficient expressed by Hounsfield units decreases in both visceral and subcutaneous fat with an increasing BMI. They also managed to show that a lower density of fat was correlated with an increased prevalence of cardiovascular risk factors. This relationship has proved to be the strongest in respect of visceral fat in women, but was also important for the subcutaneous fat in men. Additionally, there was evidence for an interaction between the density and volume of fat in both women and men [27]. We recommend including this parameter, in addition to typical morphometry, in further studies on ITF.

A separate paragraph is required to discuss problems we encountered as we attempted to compare our results with other authors [28]. Firstly, the pericardial fat nomenclature found in the literature is ambiguous [29]. Anatomically, the pericardium is a double-layered, fibro-serous sac which covers the heart from the outside. The visceral pericardium layer (being a part of epicardium) is adjacent to the surface of the cardiac muscle and is separated from it by a layer of epicardial fat. The parietal pericardium is closely fused with the fibrous pericardium, which forms the outer surface of the entire pericardium. The pericardial sac is covered from the outside by mediastinal fat, while the space between visceral pericardium and parietal pericardium (pericardial cavity) is only a potential space, containing trace amounts of fluid. Thus, the term "pericardial adipose tissue" [23,24,30-33] in our opinion is vague. It simply does not determine precisely which fat depot is referred to: whether on the outside of the pericardial sac, inside the pericardium (between the visceral layer of the pericardium and the surface of myocardium), or both of these fat clusters? Unfortunately, we realized that there is disagreement in the anatomical nomenclature of fat that surrounds the heart, its components and their locations. For example, some authors define the fat contained within the pericardium with the term "pericardial fat" [23,30-32]. For the same fat depot, other researchers have applied the term "epicardial fat" $[21,24,26,34]$. This is clearly a problem because in some papers, PAT is considered as a combination of epicardial adipose tissue and fat located outside of the pericardium $[24,35,36]$, or only as extra-pericardial fat $[7,33]$. Similarly, the fat tissue located outside the pericardium was referred to as paracardial [24,36], extrapericardial [34,37], pericardial [33], or ITF [23]. The combination of epicardial fat and extra-pericardial adipose tissue was called, for example, the pericardial [24], thoracic [34,37] and ITF [30]. As a result, the full spectrum of varying and inconsistent terminology may be encountered in the literature. Consequently, it makes it difficult to compare the results between different studies and may introduce a lot of confusion, which leads to lively discussions between authors applying different terminologies $[33,36]$.

The situation is also complicated by the fact that, in cardiac imaging methods (CT, MRI, echocardiography), the individual layers of fat are not always clearly distinguishable from one another - especially in lean people. However, data from the MESA used in the Jackson Heart Study and the work of Greif et al. [24] showed that the amount of epicardial adipose tissue correlates with the total amount of epicardial and extra-pericardial fat. Moreover, as Liu already pointed out, emerging reports seem to show that the two layers of adipose tissue are of equal metabolic activity [36]. Therefore, accurately distinguish- 
ing both types of para-cardiac fat might not be so significant in order to determine their impact on other factors (e.g. atherosclerotic process).

Another aspect that should be taken into account, when comparing the results of different studies, is the lack of consensus between authors on the methodology [28]. For example, in order to extract adipose tissue for quantitative analysis in CT, it is necessary to select a specific range of densities. Different authors use slightly different ranges: Nafakhi used the density range between -20 and $-250 \mathrm{HU}$ [31], Rosito and Mahabadi between -195 and $-45 \mathrm{HU}[23,30]$, Yong between -200 and -30 HU [26], Liu between -190 and $-30 \mathrm{HU}$ [6], Greif between -250 and $-30 \mathrm{HU}$ [24]. Moreover, some authors used cross-axial sections to measure the volume of para-cardiac fat $[24,26]$, while some others used oblique reconstructions [21]. The slice thickness and their range also differ between authors. These methodological differences can, of course, translate into differences in the measured amounts of para-cardiac adipose tissue in different works, and substantially reduce the possibility of a reliable comparison between their results. Therefore, in order to avoid mistakes and to allow comparisons between studies, an attempt to establish a consensus on the applicable nomenclature and research methodology should be made in the near future.

\section{Conclusions}

An increased chest soft tissue thickness but not an increased intra-thoracic adipose tissue volume was associated with morbid obesity. Since the quantity of the pericardial fat is not directly related to obesity, its accumulation may be related to a mechanism different than that seen in the case of subcutaneous adipose tissue growth.

\section{Acknowledgments}

The authors would like to thank Prof. Stanisław Dąbrowiecki, MD, PhD; Andrzej Gniłka, MD, PhD; and Wojciech Szczęsny, MD, PhD, for their contribution to the successful gathering of the study group.

\section{Ethical issues}

The study protocol was approved by the Bioethical Committee of the Collegium Medicum in Bydgoszcz, Nicolaus Copernicus University in Torun (consent numbers: $\mathrm{KB} / 547 / 2007$ and $\mathrm{KB} / 74 / 2014$ ). All subjects belonging to the study group received written information about the study and gave their written consent.

\section{Conflict of interest}

The authors report no conflict of interest.

\section{References}

1. Obesity and overweight. WHO Fact sheet $\mathrm{N}^{\circ} 311$ [Internet]. [cited 2016 Aug 6]. Available from: http://www.who.int/mediacentre/ factsheets/fs311/en/

2. World Health Organization: Obesity: Preventing and managing the global epidemic. Report of a WHO consultation. World Health Organ Tech Rep Ser. 2000; 894: I-XII, 1-253.

3. Lee CD, Jacobs DR, Schreiner PJ, et al. Abdominal obesity and coronary artery calcification in young adults: The Coronary Artery Risk Development in Young Adults (CARDIA) Study. Am J Clin Nutr 2007; 86: 48-54.

4. Lakka TA, Lakka HM, Salonen R, et al. Abdominal obesity is associated with accelerated progression of carotid atherosclerosis in men. Atherosclerosis 2001; 154: 497-504.

5. McClain J, Hsu F, Brown E, et al. Pericardial adipose tissue and coronary artery calcification in the Multi-ethnic Study of Atherosclerosis (MESA). Obes (Silver Spring) 2013; 21: 1056-1063.

6. Liu J, Fox CS, Hickson D, et al. Pericardial adipose tissue, atherosclerosis, and cardiovascular disease risk factors: the Jackson heart study. Diabetes Care 2010; 33: 1635-1639.

7. Iacobellis G, Corradi D, Sharma AM. Epicardial adipose tissue: Anatomic, biomolecular and clinical relationships with the heart. Nat Clin Pract Cardiovasc Med 2005; 2: 536-543.
8. Liu J, Fox CS, Hickson DA, et al. Impact of abdominal visceral and subcutaneous adipose tissue on cardiometabolic risk factors: The Jackson Heart Study. J Clin Endocrinol Metab 2010; 95: 54195426.

9. Neeland IJ, Ayers CR, Rohatgi AK, et al. Associations of visceral and abdominal subcutaneous adipose tissue with markers of cardiac and metabolic risk in obese adults. Obes (Silver Spring) 2013;21: E439-447.

10. Mohammed BS, Cohen S, Reeds D, et al. Long-term effects of large-volume liposuction on metabolic risk factors for coronary heart disease. Obesity (Silver Spring) 2008; 16: 2648-2651.

11. Patel P, Abate N. Role of subcutaneous adipose tissue in the pathogenesis of insulin resistance. J Obes 2013; 2013: 489187.

12. Niedziela J, Hudzik B, Niedziela N, et al. The obesity paradox in acute coronary syndrome: A meta-analysis. Eur J Epidemiol 2014; 29: 801-812.

13. Narumi H, Yoshida K, Hashimoto N, et al. Increased subcutaneous fat accumulation has a protective role against subclinical atherosclerosis in asymptomatic subjects undergoing general health screening. Int J Cardiol 2009; 135: 150-155.

14. Serafin Z, Rusak G, Strześniewski P, et al. Comparison of calcium scoring with 4-Multidetector Computed Tomography (4-MDCT) 
and 64-MDCT: A phantom study. J Comput Assist Tomogr 2012; 36: 88-93.

15. Serafin Z, Lasek W, Laskowska K. Phantom-calibrated versus automatic coronary artery mass quantification with multidetector-row computed tomography: In vitro and in vivo study. Acta Radiol 2008; 49: 1007-1015.

16. Fox CS, Massaro JM, Hoffmann U, et al. Abdominal visceral and subcutaneous adipose tissue compartments: Association with metabolic risk factors in the Framingham Heart Study. Circulation 2007; 116: $39-48$

17. Miranda Prado C. Evaluation of visceral and subcutaneous fat by ultrasound and its relationship with clinical and metabolic parameters of insulin resistance and subclinical atherosclerosis. Open J Endocr Metab Dis 2012; 2: 63-69.

18. Iacobellis G, Ribaudo MC, Assael F, et al. Echocardiographic epicardial adipose tissue is related to anthropometric and clinical parameters of metabolic syndrome: A new indicator of cardiovascular risk. J Clin Endocrinol Metab 2003; 88: 5163-5168.

19. Iacobellis G, Leonetti F. Epicardial adipose tissue and insulin resistance in obese subjects. J Clin Endocrinol Metab 2005; 90: 6300-6302.

20. Jeong J-W, Jeong MH, Yun $\mathrm{KH}$, et al. Echocardiographic epicardial fat thickness and coronary artery disease. Circ J 2007; 71: 536-539.

21. Gorter PM, van Lindert AS, de Vos AM, et al. Quantification of epicardial and peri-coronary fat using cardiac computed tomography; reproducibility and relation with obesity and metabolic syndrome in patients suspected of coronary artery disease. Atherosclerosis 2008; 197: 896-903.

22. Pang C, Gao Z, Yin J, et al. Macrophage infiltration into adipose tissue may promote angiogenesis for adipose tissue remodeling in obesity. Am J Physiol Endocrinol Metab 2008; 295: E313-322.

23. Mahabadi A, Massaro JM, Rosito G, et al. Association of pericardial fat, intrathoracic fat, and visceral abdominal fat with cardiovascular disease burden: The Framingham Heart Study. Eur Heart J 2009; 30: 850-856.

24. Greif M, Becker A, Von Ziegler F, et al. Pericardial adipose tissue determined by dual source CT is a risk factor for coronary atherosclerosis. Arterioscler Thromb Vasc Biol 2009; 29: 781-786.

25. Thanassoulis G, Massaro JM, Hoffmann U, et al. Prevalence, distribution, and risk factor correlates of high pericardial and intrathoracic fat depots in the Framingham heart study. Circ Cardiovasc Imaging 2010; 3: 559-566.
26. Yong HS, Kim EJ, Seo HS, et al. Pericardial fat is more abundant in patients with coronary atherosclerosis and even in the non-obese patients: Evaluation with cardiac CT angiography. Int J Cardiovasc Imaging 2010; 26 (Suppl 1): 53-62.

27. Rosenquist KJ, Pedley A, Massaro JM, et al. Visceral and subcutaneous fat quality and cardiometabolic risk. JACC Cardiovasc Imaging 2013; 6: 762-771.

28. Sardanelli F, Bashir H, Berzaczy D, et al. The role of imaging specialists as authors of systematic reviews on diagnostic and interventional imaging and its impact on scientific quality: Report from the EuroAIM Evidence-based Radiology Working Group. Radiology 2014; 272: 533-540.

29. Talman AH, Psaltis PJ, Cameron JD, et al. Epicardial adipose tissue: Far more than a fat depot. Cardiovasc Diagn Ther 2014; 4: 416-429.

30. Rosito G, Massaro JM, Hoffmann U, et al. Pericardial fat, visceral abdominal fat, cardiovascular disease risk factors, and vascular calcification in a community-based sample the framingham heart study. Circulation 2008; 117: 605-613.

31. Nafakhi H, Al-Mosawi A, Al-Nafakh H, Tawfeeq N. Association of pericardial fat volume with coronary atherosclerotic disease assessed by CT angiography. Br J Radiol 2014; 87: 20130713.

32. Ding J, Kritchevsky SB, Harris TB, et al. The association of pericardial fat with calcified coronary plaque. Obesity (Silver Spring) 2008; 16: 1914-1919.

33. Iacobellis G, Malavazos A. Pericardial adipose tissue, atherosclerosis, and cardiovascular disease risk factors: The Jackson Heart Study: Comment on Liu et al. Diabetes Care 2010; 33: e127.

34. Dey D, Nakazato R, Li D, et al. Epicardial and thoracic fat - Noninvasive measurement and clinical implications. Cardiovasc Diagn Ther 2012; 2: 85-93.

35. Wheeler GL, Shi R, Beck SR, et al. Pericardial and visceral adipose tissues measured volumetrically with computed tomography are highly associated in type 2 diabetic families. Invest Radiol 2005; 40: 97-101.

36. Liu J, Taylor H, Fox CS, et al. Pericardial Adipose Tissue, Atherosclerosis, and Cardiovascular Disease Risk Factors: The Jackson Heart Study: Response to Iacobellis and Malavazos. Diabetes Care 2010; 33: e128.

37. Tamarappoo B, Dey D, Shmilovich H, et al. Increased pericardial fat volume measured from noncontrast CT predicts myocardial ischemia by SPECT. JACC Cardiovasc Imaging 2010; 3: 1104-1112. 